\section{A Case of False Positive Macro-prolactin}

Sir,

A 31-year, non-smoker, Caucasian lady was referred by her general practitioner (GP) to Endocrinology Clinic with hyperprolactinaemia. She denied galactorrhoea, irregular periods and visual problems. She complained of headaches for previous 10 years with increase in frequency recently. She denied clinical features of other pituitary hormones' excess or deficiency. She was receiving treatment for acne for previous few years from her dermatologist. She used doxycycline for six months last year and was now using isotretinoin for a few months for the same. She noticed significant clinical improvement in her acne. Her mother had type 2 diabetes and paternal uncle had essential hypertension. She was allergic to penicillin. On examination, her vital signs were within normal ranges, visual fields were full on confrontation method and no neurological signs were detected. Laboratory investigations showed normal pituitary profile except high total prolactin, $2055 \mathrm{mIU} / \mathrm{L}$ (Normal range: 102-496 mIU/L) and a positive macro-prolactin screen using polyethylene glycol (PEG) with bioactive prolactin, $332 \mathrm{mIU} / \mathrm{L}(<350 \mathrm{mIU} / \mathrm{L})$. Since the bioactive prolactin was within normal range and she was asymptomatic, no treatment was required, so was discharged from clinic back to her GPcare.

Tablel:

\begin{tabular}{|c|c|c|c|c|}
\hline & Time 0 & 15 months & 17 months & 18 months \\
\hline Prolactin (mIU/L) & $\begin{array}{c}2055 \\
\text { PEG* }\end{array}$ & $\begin{array}{l}2017 \\
\text { PEG }\end{array}$ & $\begin{array}{r}1913 \\
\text { PEG }\end{array}$ & $\begin{array}{c}3025 \\
\text { Gel filtration }\end{array}$ \\
\hline $\begin{array}{l}\text { Macroprolactin/ } \\
\text { bioactive }\end{array}$ & $\begin{array}{l}\text { Predominantly } \\
\text { macroprolactin }\end{array}$ & $\begin{array}{c}\text { Predominantly } \\
\text { bioactive }\end{array}$ & $\begin{array}{l}\text { Predominantly } \\
\text { bioactive }\end{array}$ & $\begin{array}{l}\text { Predominantly } \\
\text { bioactive }\end{array}$ \\
\hline LH (IU/L) & 1.1 & & 1.2 & \\
\hline FSH (IU/L) & 2.6 & & 2.3 & \\
\hline TSH $(0.27-4.27 \mathrm{mlU} / \mathrm{L})$ & 3.45 & & 2.94 & \\
\hline $\mathrm{FT}_{4}(12-22 \mathrm{pmol} / \mathrm{L})$ & 11.6 & & 12.3 & \\
\hline $\begin{array}{l}\text { Cortisol (176-536 } \\
\text { nmol/L) }\end{array}$ & 218 & & 438 & \\
\hline $\begin{array}{l}\text { Oestradiol } \\
\text { (Follicular phase } \\
45-854, \\
\text { Ovulatory phase } \\
151 \text { - } 1461, \\
\text { Luteal phase } \\
82-1251 \text { ) }\end{array}$ & 468 & & 426 & \\
\hline IGF-1 (15-42 nmol/L) & 25.6 & & 18.1 & \\
\hline
\end{tabular}

About 12 months later, her GP repeated her blood tests including prolactin levels. Her total prolactin remained high at $2017 \mathrm{mIU} / \mathrm{L}$ with minimal macro-prolactin detected using PEG. The test was repeated a month later, which once again had bioactive prolactin of $1913 \mathrm{mIU} / \mathrm{L}$ (Table I). We then performed the test using gel filtration, which confirmed total prolactin of $3025 \mathrm{mIU} / \mathrm{L}$, predominantly bioactive. Going through her history, she discontinued isotretinoin about nine months ago. There was no other change compared to a year ago, when her first prolactin level was measured. Her pituitary MRI scan detected a micro-prolactinoma of $4 \mathrm{~mm}$ and was then treated with cabergoline, as she was planning to conceive. Her prolactin responded well to this treatment and started to settle.

Macro-prolactin, also called "big-big" prolactin, is formed by prolactin-immunoglobulin complexes and may cause elevation of serum prolactin concentrations measured by standard assays, potentially leading to unnecessary investigations and/or treatment. $^{1-3}$

This case of false positive macro-prolactin most likely was due to high immunoglobulin levels with isotretinoin use for acne in a young female. Isotretinoin should be considered a possible cause of false positive macro-prolactin in selected patients with high macro-prolactin levels.

\section{PATIENT'S CONSENT:}

Informed consent was obtained from the patient to publish the data concerning this case.

\section{CONFLICT OF INTEREST:}

The author declared no conflict of interest.

\section{AUTHOR'S CONTRIBUTION:}

AIS: Responsible for all aspects of this manuscript.

\section{REFERENCES}

1. Siddiqi A. See your endocrinologist for a pituitary tumour; you may not need surgery. I Cancer Applied Spec 2017; 3(1):2.

2. Ram S, Harris B, Fernando JJ, Gama R, Fahie-Wilson M. False-positive polyethylene glycol precipitation tests for macroprolactin due to increased serum globulins. Ann Clin Biochem 2008; 45(Pt3): 256-9. doi: 10.1258/acb.2008. 007233.

3. Coble BI, Dahlgren C, Molin L, Stendahl O. Neutrophil function in psoriasis; Effects of retinoids. Acta Derm Venereol 1987; 67(6):481-90. doi: 10.2340/00015555 67481490 .

Ahmed Imran Siddiqi

Department of Medicine, Shaukat Khanum Memorial Cancer Hospital and Research Centre, Lahore, Pakistan

Correspondence to: Dr. Ahmed Imran Siddiqi, Department of Medicine, Shaukat Khanum Memorial Cancer Hospital and Research Centre, Lahore, Pakistan

E-mail: ahmed.siddiqi@nhs.net

Received: June 09, 2020; Revised: November 19, 2020; Accepted: December 05, 2020

DOI: https://doi.org/10.29271/jcpsp.2021.06.749 